\title{
EL MANEJO DE LOS PROBLEMAS DE SALUD MENTAL POR PARTE DE LOS MÉDICOS
}

Luis Carlos Taborda R. MD*

\section{Introducción}

Es importante reconocer que los avances psicofarmacoterapéuticos que se han dado para el manejo de los problemas de salud mental, no han repercutido de manera favorable en la atención de la población por diversos aspectos que bien vale la pena considerar, muchos de los cuales podemos y debemos corregir porque se encuentran dentro de nuestro espacio de acción y responsabilidad profesional, ética y social.

Bien conocido es para todos que cerca del $90 \%$ de los pacientes que llegan a la consulta de especialistas en psiquiatría, son remitidos después de haber sido tratados en forma inadecuada (y, por qué no decir "maltratados"), por nuestros colegas médicos generales y profesionales de otras especialidades: subdosificados, por supuesto con malos resultados y vueltos a formular y de nuevo subdosificados con uno, dos o tres antidepresivos de diferentes grupos, cambiándolos cada ocho a quince días o de acuerdo con la frecuencia de las consultas. Además con varios ansiolíticos, inclusive con el riesgo de crear dependencia en personas jóvenes que pueden cursar con dificultades emocionales a largo plazo. Todo esto constituye un círculo vicioso que lleva a que el paciente nos llegue con una carga de desconfianza, para tener que ser reeducado en un nuevo esquema terapéutico y en la mayoría de los casos no solo el sino la familia han tenido que hacer importantes esfuerzos económicos, afectivos y sociales, sin ver una respuesta positiva para la salud de su pariente.

¿Qué responsabilidad tenemos nosotros, profesores universitarios, en esto? La Organización Mundial

* Profesor Titular de Psiquiatría, Fundación Universitaria de Ciencias de la Salud. Miembro Honorario de la Sociedad de Cirugía de Bogotá, Colombia.

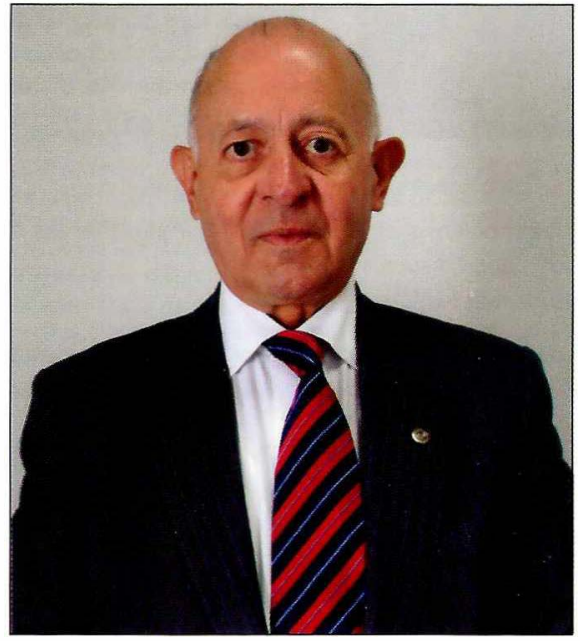

de la Salud estima que por lo menos 500 millones de personas sufren de algún tipo de enfermedad mental, a pesar de que con frecuencia estos problemas no se detectan o no se tratan; por esto, los trabajadores de la salud deben considerar los estados de salud mental y física de cada persona como un todo y que hagan de la primera una parte integral de la atención primaria en salud, sobre lo cual ha insistido el profesor Jorge Alberto Costa e Silva, director del Departamento de Salud Mental y Prevención del Abuso de Drogas de la Organización Mundial de la Salud. ${ }^{1}$

\section{El problema de la educación médica}

Para Quintero ${ }^{2}$, los cursos de la educación médica de pregrado están compuestos por varias disciplinas que comprenden ciencias básicas, biomédicas, sociohumanistas, del comportamiento y las clínicas. Ha sido tradicional que el currículo haya sido diseñado por separado, haciendo énfasis en las ciencias básicas y biomédicas en los años iníciales y en la clínicas en la fase avanzada. Al estudiante se le pide, sin embargo, 
que integre esos conocimientos durante sus años de práctica y después de graduado, sin tener en cuenta que con tal división no se presentan ni los enfermos ni las enfermedades.

A finales del siglo pasado se introdujo el concepto de integración curricular para permitir que las diferentes disciplinas se presenten de una manera combinada y organizada a través de experiencias de aprendizaje, integradas en forma tal que el estudiante pueda, a través de ellas, desarrollar competencias cognoscitivas y habilidades sustentadas en el aprendizaje basado en casos o problemas, que es la forma como ocurren las enfermedades. Este enfoque difiere de la forma clásica de enseñanza-aprendizaje, en la cual las disciplinas se presentan de forma desintegrada y se le deja al estudiante la carga de hacer su integración.

A pesar de que esta metodología está bien definida y aceptada por la mayoría de los programas de medicina de pregrado, su implementación varía mucho, pues existen por lo menos once grados de integración, como ha sido señalado por $\operatorname{Harden}^{3}$ que van desde enseñar la disciplina en forma aislada, como es usual en un currículo carente de integración, hasta la forma más integradora de todas, que es el currículo interdisciplinario y transdisciplinario.

La interdisciplinariedad es "el estudio de un fenómeno que involucra dos o más disciplinas simultáneamente", 4 e implica un alto grado de integración. Se pierde la perspectiva asignaturista y así los contenidos de conocimientos y su extensión no están demarcados entre sí. La organización sistémica es la mejor forma de llevar a cabo la enseñanza interdisciplinaria.

La transdiciplinariedad, por su parte, es un proceso mental del individuo para construir significados (comprensión) de la información y del conocimiento adquirido, y depende de su propia percepción. ${ }^{5}$ A ella se llega solo si existe la interdisciplinariedad en el currículo; de ahí su importancia.

Sin embargo, continua Quintero, las reformas en la enseñanza y aprendizaje de la medicina continúan basándose en una visión biologista del proceso salud-enfermedad; es necesario incorporar de manera integrada también, las ciencias socio-humanistas y de salud mental de poblaciones, pues las acciones que se aprecian en ese sentido se han limitado en algunos casos a introducir algunas asignaturas aisladas como la antropología, la sociología, la psicología o la salud comunitaria entre otras, sin ninguna articulación sistémica con el resto del currículo o, en otros, a incorporarlas como ciencias auxiliares de la salud pública o de la medicina preventiva, para dar cuenta de los factores considerados como externos o socioambientales, dentro del modelo de los "factores de riesgo".

La razón para implementar un currículo integrado no es otra que facilitar la Enseñanza para la Comprensión (EpC), entendida en términos de cómo hacer cosas usando los conocimientos previos para resolver nuevos problemas en situaciones inéditas ${ }^{6}$, pues como bien decía Llinás?: "Los procesos educativos están estructurados sobre el saber, que es conocer muchas cosas. Debería ser sobre el entender, es decir poner todo en contexto. Los datos sueltos no sirven para nada".

Henry J. Walton de la Universidad de Edimburgo, expresidente de la Federación Mundial de Educación Médica conceptúa que "en la actualidad, los programas de enseñanza en medicina, deben enfocar también la prevención de la enfermedad y la promoción de la salud, temas a los cuales se les brinda un reconocimiento únicamente marginal en los programas convencionales". ${ }^{8}$

Sabemos a ciencia cierta que alrededor de $15 \%$ de los pacientes ambulatorios con diagnóstico médico que se atienden por consulta externa presentan trastornos emocionales asociados y este porcentaje llega a $20 \mathrm{o}$ $30 \%$ en la población sin diagnóstico médico. Por estas razones, todo futuro médico debe tener el conocimiento de estos padecimientos y la capacidad para atenderlos en forma adecuada; es una de las razones por la cual la enseñanza debe abordar los trastornos emocionales de cada una de las especialidades en atención primaria, en hospitales generales y en centros comunitarios. Por ejemplo, cuando un estudiante está rotando por urología debe tener la oportunidad de recibir la docencia adecuada 
de las dificultades emocionales que sufre un paciente que ha sido sometido a prostactetomía al que no le han explicado que no va a volver a presentar eyaculación externa; hay que preguntarle cómo está su vida sexual y brindarle todas las explicaciones necesarias para que vuelva a tener una buena funcionalidad. En ginecología todavía hay quienes consideran que la depresión posparto es algo normal. La altísima frecuencia de trastornos depresivos y ansiosos posteriores a un infarto de miocardio o luego de una cirugía cardiovascular, que muchas veces nos llegan en forma indirecta a consultar si pueden o no tener una vida sexual normal. Todos los cuadros depresivos que se ven con posterioridad a los accidentes cerebrales, que cuando ocurren en el hemisferio dominante o hacia el polo anterior son más tempranas y severas, independiente del tamaño de la hemorragia, mientras que la depresión en casos de hemorragias en hemisferio no dominante son de manifestación tardía y se pueden confundir con cuadros de deterioro cerebral (mucho más grave constatar que algunos especialistas las califican como normales por las secuelas neurológicas que presentan). Aquí podríamos hacer una enumeración interminable, perono quiero dejar pasar inadvertidas todas las implicaciones emocionales del dolor crónico, en las cuales encontramos la triada al asociarse con depresión e insomnio. Esta es una de las razones fundamentales para que en todo equipo interdisciplinario de clínica del dolor, por ejemplo, haya un psiquiatra. Es preciso insistir en que en muchos de estos casos no solo se deben examinar a los pacientes, sino también entrevistar a los familiares, amigos o acudientes, para conocer el estado emocional del paciente en su entorno y las dificultades que pueda tener el grupo familiar, laboral o social. Debemos recordar que todo grupo funciona como un "móvil" (muñequitos que se ponen sobre las cunas de los niños), de modo que al halar un muñeco, todos se mueven en mayor o en menor proporción.

Estos programas deben tener una autoevaluación semestral en todas y cada una de las rotaciones. En ellas deben participar docentes y estudiantes; una vez calificados los resultados del programa, se harán recomendaciones para ponerlas en práctica en el próximo semestre y así mantener los programas siempre actualizados. Todo lo anterior es la forma como proponemos que se debe hacer la docencia de pregrado en el campo de salud mental y no limitarla a la práctica especializada en psiquiatría, con pacientes hospitalizados y muchos de ellos crónicos a quienes los médicos generales y de otras especialidades no van a tener la oportunidad de ver en su ejercicio profesional. En esta misma línea se presenta el Proyecto de Programa Global de la Federación Mundial de Educación Médica. ${ }^{9}$

Sin duda, el énfasis de la educación de pregrado en psiquiatría debe estar focalizado en la promoción de la salud mental y en la prevención de la enfermedad mental.

Para Beddington, Cooper, Field, y col..$^{9}$ los países tienen que aprender a capitalizar los recursos cognitivos de sus ciudadanos si quieren prosperidad económica y social, y esto implica entre otros aspectos la detección temprana de la enfermedad mental y la prevención de la misma, lo cual hace necesario que los currículos cambien la forma con la cual están preparando a los futuros médicos. Es lo que se conoce como el "mejoramiento del capital mental de las naciones", entendiéndolo bajo dos perspectivas, los recursos cognoscitivos y los emocionales que incluyen no solo la habilidad cognoscitiva de la gente sino también la flexibilidad y la eficiencia para aprender, la inteligencia emocional, el uso de herramientas sociales y los factores de protección para enfrentar situaciones complejas. El futuro médico debe además tener esas capacidades cuando ingresa a la universidad.

Entonces, si queremos tener médicos generales integrales en el futuro, debemos afinar nuestros procesos de selección, analizando y estudiando los datos biográficos del aspirante, las calificaciones de bachillerato, la verificación de las referencias, la aplicación de baterías de las pruebas psicológicas y la conformación de equipos de entrevistadores capaces de calificar la motivación, el compromiso social, la estabilidad afectiva, el interés, el conocimiento de la carrera y la institución a donde solicita ingresar. Estos mismos entrevistadores deberán realizar los procesos de seguimiento durante toda la carrera. 